\title{
Coffee intake mitigated inflammation and obesity-induced insulin resistance in skeletal muscle of high-fat diet-induced obese mice
}

\author{
Huijuan Jia • Wanping Aw $\cdot$ Kenji Egashira \\ Shoko Takahashi $\cdot$ Shinya Aoyama \\ Kenji Saito $\cdot$ Yoshimi Kishimoto $\cdot$ Hisanori Kato
}

Received: 17 November 2013/Accepted: 17 February 2014/Published online: 6 March 2014

(C) Springer-Verlag Berlin Heidelberg 2014

\begin{abstract}
Epidemiologic findings offer the promise that coffee or its many constituents may be useful as a dietary intervention in type 2 diabetes (T2D) prevention. We aimed to elucidate the molecular mechanisms involved in the ameliorative effects of caffeinated coffee (CC), decaffeinated coffee (DC) and unroasted caffeinated green coffee (GC) on skeletal muscle gene expression profiles and their relationships in an obesity animal model. Eight-weekold male C57BL6 mice were raised for 9 weeks ad libitum on a normal diet, a high-fat diet, or high-fat diet containing $2 \%$ freeze-dried CC, or DC, or GC. Total RNA and protein were extracted from skeletal muscle and subjected to
\end{abstract}

Huijuan Jia and Wanping Aw have contributed equally to this work.

Electronic supplementary material The online version of this article (doi:10.1007/s12263-014-0389-3) contains supplementary material, which is available to authorized users.

H. Jia $\cdot$ K. Saito $\cdot$ H. Kato $(\bowtie)$

Corporate Sponsored Research Program "Food for Life,"

Organization for Interdisciplinary Research Projects,

The University of Tokyo, 1-1-1, Yayoi, Bunkyo-ku,

Tokyo 113-8657, Japan

e-mail: akatoq@mail.ecc.u-tokyo.ac.jp

W. Aw

Graduate School of Biomedical Science, Tokyo Medical and Dental University, Bunkyo-ku, Tokyo, Japan

K. Egashira $\cdot$ S. Takahashi $\cdot$ S. Aoyama $\cdot$ H. Kato Department of Applied Biological Chemistry,

Graduate School of Agricultural and Life Sciences,

The University of Tokyo, Bunkyo-ku, Tokyo, Japan

Y. Kishimoto

Institute of Environmental Science for Human Life,

Ochanomizu University, Bunkyo-ku, Tokyo, Japan microarray (Mouse Genome 430 2.0, Affymetrix) and western blotting analyses, respectively. Coffee intake mitigated the insulin resistance by decreasing plasma glucose levels during an insulin tolerance test and by increasing tyrosine phosphorylation of insulin receptor substrate-1 (IRS-1), p85/IRS-1 complex and pAkt/PKB (protein kinase B). In addition, coffee intake down-regulated the antiinflammatory genes activating transcription factor 3, FBJ osteosarcoma oncogene, heat shock protein $1 \mathrm{~A}$, heat shock protein $1 \mathrm{~B}$ and synuclein, gamma and the inflammationassociated insulin signaling genes stearoyl-coenzyme A desaturase 1 and secreted phosphoprotein 1 . These results provide scientific insight on the probable positive effects of coffee intake on impaired insulin signaling, inflammation and obesity, thereby providing a new perspective on the prevention of obesity and T2D.

Keywords Coffee intake - Insulin resistance · Inflammation and obesity - Skeletal muscle . Transcriptomic analysis $\cdot$ High-fat diet

\section{Introduction}

Coffee consumption is a trend that presumably originated in northeast Africa, spread across the Middle East in the fifteenth century and then to Europe (Gray 1998), and now, approximately 2.25 billion cups of coffee are consumed across the globe every day (Stefano 2002). Epidemiologic findings offer the promise that coffee, or one or more of its many constituents, may prove to be useful as a novel approach for type 2 diabetes (T2D) prevention (Hu et al. 2006; Paynter et al. 2006). The number of people estimated to develop T2D is rapidly increasing, and this is strongly associated with the changes in dietary habits. As such, it is 
of considerable importance to identify possible dietary interventions for the primary prevention of T2D.

Coffee is a complex mixture of hundreds of chemicals that occur naturally or are formed during the roasting process. Caffeic acid (CA) occurs in food mainly as esters known as chlorogenic acids (CGAs) (Rice-Evans et al. 1996). Roasting markedly affects the composition of coffee, and compounds with antioxidant properties (mainly CGAs) are lost during the roasting of coffee beans (Abebe and Kebba 2013). In a db/db diabetic mouse model, CA also exhibited anti-diabetic properties by markedly increasing glucokinase activity and simultaneously lowering glucose-6-phosphatase and phosphoenolpyruvate carboxykinase activities in the liver (Jung 2006). CGA has been reported to modulate the glucose-6-phosphatase involved in glucose metabolism and to reduce the risk of cardiovascular disease by decreasing the oxidation of lowdensity lipoprotein cholesterol and total cholesterol (TC) (Nardini et al. 1995). In diet-induced obese mice, CGAs also altered the plasma adipokine level and body fat distribution and down-regulated fatty acid and cholesterol biosynthesis, but they up-regulated fatty acid oxidation and hepatic peroxisome proliferator-activated receptor alpha expression (Cho et al. 2010).

In addition to the constituents of coffee, coffee itself has been reported to down-regulate the expression of inflammatory cytokines [i.e., monocyte chemotactic protein-1, interleukin (IL)-1 $\beta$, IL-6, and leptin] in adipose tissue and liver (Matsuda et al. 2011), and to increase the insulininduced serine phosphorylation of Akt/PKB (protein kinase $\mathrm{B}$ ) in the liver and skeletal muscle of diabetic mice (Kobayashi et al. 2012). As highlighted above, most studies have focused on the effects of coffee intake on the liver and adipose tissue. The influence of coffee intake on the expression of inflammatory cytokines and the molecular mechanisms underlying the improved insulin sensitivity in skeletal muscle are relatively unknown, despite the fact that skeletal muscle is a fundamental player in glucose metabolism and homeostasis in humans, accounting for nearly $40 \%$ of body mass (DeFronzo et al. 1992).

In recent years, applications of microarray technology, a robust tool in the global evaluation of gene expression profiles, have provided vital information regarding the use of nutraceuticals for the prevention and management of several human diseases (Jia et al. 2013a, b). In the present study, we used DNA microarray and western blotting analysis to investigate the influence of the intake of different types of coffee-caffeinated coffee (CC), decaffeinated coffee (DC), and unroasted caffeinated green coffee (GC)—on skeletal muscle gene expression profiles and their relationships in a high-fat diet-induced obesity animal model. We aimed to elucidate the molecular mechanisms involved in the ameliorative effects of coffee intake on impaired insulin signaling, inflammation and obesity, thereby providing new perspectives and scientific evidence to support the effectiveness of coffee intake in controlling obesity and T2D.

\section{Materials and methods}

Animals and dietary treatment

Seven-week-old male C57BL6J mice obtained from Charles River Japan (Tokyo) were housed individually in animal cages in a room with controlled temperature $\left(23 \pm 2{ }^{\circ} \mathrm{C}\right)$, humidity $(50 \pm 10 \%)$, and lighting (lights on from 0800 to 2000). After 7 days of acclimatization, the mice were divided into five groups with equal mean body weights. The normal diet group was fed D12450B (ND; $10 \% \mathrm{kcal}$ fat), the high-fat diet group was fed D12492 (HF; $60 \% \mathrm{kcal}$ fat), and the three coffee groups were fed the high-fat diet containing $2 \%$ CC (HFCC), or DC (HFDC), or GC (HFGC), respectively. Freeze-dried coffee (Takahashi et al. 2014) provided by Nestle Japan Ltd. (Tokyo) were mixed into the diets by Research Diets Inc. (New Brunswick, NJ, USA) (Supplementary Table 1). The mice received their respective diets and drinking water ad libitum for 9 weeks. Food intake and body weights were monitored throughout the experiment.

\section{Blood collection and tissue harvesting}

Prior to sacrifice, mice were starved for $16 \mathrm{~h}$ and were deeply anaesthetized with sodium pentobarbital, followed by bleeding from the carotid artery. The gastrocnemius muscle from the lower limb, the liver, and mesenteric and epididymal adipose tissues were excised and snap-frozen in liquid nitrogen and stored at $-80{ }^{\circ} \mathrm{C}$ until further analysis. Plasma was obtained by centrifuging blood at $1,000 \mathrm{~g}$ for 15 min at $4{ }^{\circ} \mathrm{C}$. This study was approved by the Animal Care and Use Committee of The University of Tokyo, and the animals were treated in accordance with the committee's guidelines.

\section{Biochemical assays}

Triglyceride (TG) and TC concentrations in plasma were measured using a cholesterol $E$ test kit and a TG $E$ test (Wako Pure Chemical Industries, Osaka, Japan) according to the manufacturer's instructions. Total lipids in the liver were extracted according to the Folch method, after which the hepatic concentrations of TG and TC were measured by the tests mentioned above. 
DNA microarray analysis

The total skeletal muscle RNA extraction and quality assessment were conducted as described (Jia et al. 2013a). Total RNA from each respective group were pooled $(n=4-5)$. The microarray analysis (Mouse Genome 430 2.0 Array Gene Chip, Affymetrix, Santa Clara, CA, USA) was carried as out as described (Jia et al. 2013a). Array images were analyzed with Microarray Suite version 5.0 (Affymetrix). After normalizing the differences in microarray intensities, we performed pairwise comparisons between the ND (as baseline) and HF values, and between the HF values (as the baseline) and those of the HFCC or HFDC or HFGC group (Supplementary Materials). Genes were considered to be differentially expressed if they were up- or down-regulated by 1.2-fold compared to the HF group. Genes were considered to be differentially expressed if they were up-or down-regulated by 1.2 -fold compared to the HF group.

Real-time reverse transcription polymerase chain reaction

We performed a real-time reverse transcription polymerase chain reaction (RT-PCR) as described (Jia et al. 2013a) to detect the expression of genes related to inflammation, obesity, insulin resistance and T2D. Primers were designed with a Web application (PRIMER3), and their sequences are as shown in Supplementary Table 2. The relative amounts of mRNA were normalized to $\beta$-actin expression levels in each sample, and the data are presented as the fold change of normalized mRNA amounts of each sample compared to those of the ND group.

\section{Quantification of Atf3 and Socs3 protein levels}

Excised gastrocnemius muscle was minced and homogenized in $0.02 \mathrm{~mol} / \mathrm{L}$ ice-cold phosphate-buffer saline $(\mathrm{pH}$ 7.1), after which homogenates were centrifuged for $15 \mathrm{~min}$ at $1,500 \mathrm{~g}$. We measured the protein levels of Atf3 and Socs 3 in the supernatant using enzyme-linked immunosorbent assay (ELISA) kits following the manufacturer's instructions (MyBioSource, San Diego, CA, USA). Muscle protein concentrations were measured using the Bradford assay. Data were then presented as the fold change of the normalized protein concentration comparing to HF group.

\section{Insulin tolerance test}

To further investigate the effects of coffee intake on insulin signaling in the skeletal muscle, we performed an animal experiment identical to that described above in the section "Animals and Dietary Treatment." At week 8 of the experimental period, we performed an insulin tolerance test (ITT) with 4-h fasting. The solutions of bovine insulin $(0.75$ $\mathrm{U} / \mathrm{kg}$ body weight) were injected i.p. Glucose levels were determined using Ascensia BREEZE (Bayer Medical Ltd., Leverkusen, Germany) in blood obtained from the tail vein at $0,15,30,60,120$, and $150 \mathrm{~min}$ after the injection. We calculated the areas under the curve (AUC) as Glucose $\left(\mathrm{AUC}_{\mathrm{glucose}}\right)$.

In vivo insulin stimulation

At the end of the 9-week experimental period, in each group, the mice were divided again into saline $(n=4)$ and insulin $(n=5)$ groups. All mice were starved for $16 \mathrm{~h}$ and then refed for $1.5 \mathrm{~h}$ before being killed. The mice were deeply anaesthetized with sodium pentobarbital. As soon as the effect of the anesthesia was assured by the loss of pedal reflexes, the abdominal cavity was opened, and normal saline or $0.14 \mathrm{U} / 200 \mu \mathrm{L}$ of bovine insulin (Sigma-Aldrich, Saint Louis, MO, USA) was injected into the cava vein. At 2 min after the insulin administration, skeletal muscles from the lower limb were excised and snap-frozen in liquid nitrogen. The frozen tissues were homogenized in a polytron homogenizer at $4{ }^{\circ} \mathrm{C}$ with 10 times the muscle weight of homogenizing buffer (50 mM HEPES-NaOH, pH 7.6, $10 \mathrm{mM}$ sodium orthovanadate, $10 \mathrm{mM}$ sodium pyrophosphate, $100 \mathrm{mM}$ sodium fluoride, $2 \mathrm{mM}$ phenylmethylsulfonyl fluoride, $100 \mathrm{KIU} / \mathrm{mL}$ aprotinin, $2 \mathrm{mM}$ EDTA, and $2 \%$ Triton X-100). The homogenate was centrifuged at $100,000 \mathrm{~g}$ for $60 \mathrm{~min}$ at $4{ }^{\circ} \mathrm{C}$, and the supernatants were collected for the western blotting analysis. The protein concentration was determined with a Bio-Rad Protein Assay kit (Bio-Rad, Gladesville, New South Wales, Australia).

Western blotting analysis of Ser473 and Akt

Twenty microgram protein lysates were suspended in Laemmli's sample buffer, boiled for $5 \mathrm{~min}$ and used for Western blotting. Samples were subjected to SDS-PAGE on acrylamide gel, and the proteins in the gel were transferred onto PVDF membranes (Hybond P, GE Healthcare, Tokyo, Japan) and incubated overnight at $4{ }^{\circ} \mathrm{C}$ with antiphospho Akt/PKB (Ser473) (1:1,000; Cell Signalling Technology, Danvers, MA, USA), or anti-Akt (4058S, 1:1,000; Cell Signalling Technology), and then washed with Tris-buffered saline containing $0.1 \%$ Tween 20 . The membranes were then incubated with 1:5,000 diluted horseradish peroxidase-conjugated secondary antibodies diluted in TBS-T at room temperature for $1 \mathrm{~h}$, and washed again as described above. The bands of immunoreactive proteins were detected with an enhanced chemiluminescence kit (GE Healthcare, Buckinghamshire, UK) and quantified using an LAS-1000 plus (Fuji Film Co., Kanagawa, Japan). 
Immunoprecipitation of IRS-1 and western blotting analysis of IRS-1, p85, and phosphotyrosine

For immunoprecipitation of insulin receptor substrate-1 (IRS-1), the extracted protein (4 mg) was incubated with anti-IRS-1 (4 $\mu \mathrm{g})$ antibody at $4{ }^{\circ} \mathrm{C}$ overnight, followed by the addition of $30 \mu \mathrm{l}$ protein G-Sepharose $4 \mathrm{FF}(50 \% \mathrm{v} / \mathrm{v})$ for $1.5 \mathrm{~h}$. The immunocomplexes were washed three times with the homogenizing buffer at $4{ }^{\circ} \mathrm{C}$, suspended in Laemmli's sample buffer, and boiled for $5 \mathrm{~min}$. Subsequent western blotting analysis using anti-IRS-1 (06-248, 1:1,000; Millpore, Billerica, MA, USA), or anti-phosphotyrosine (4G10, 1:1,000; Millpore), or anti-p85 (1:1,000; Millpore) was performed as described above.

\section{Statistical analysis}

Data are presented as mean values \pm standard error (SE). The statistical analysis was conducted using a one-way analysis of variance, and significant differences in mean values were evaluated with Dunnett's test at the level of $p<0.05$ versus the HF group.

\section{Results}

\section{General characteristics}

There was no significant difference in food intake among the five groups, although the ND group had a lower calorie intake. At the time of dissection, the HF group's body weight, liver weight, mesenteric, and epididymal fat weight values were significantly higher than those of the ND group. The levels of plasma TG and hepatic TC were significantly higher, and the plasma TC and hepatic TG levels tended to be higher in the HF group compared with the ND group (Table 1).
Mice in the HFCC, HFDC, and HFGC groups gained significantly less weight as compared with mice in $\mathrm{HF}$ group. The DC significantly suppressed the increase in mesenteric fat weight, whereas the apparent suppression observed in CC and GC was not statistically significant. The epididymal fat weights tended to be lower in all coffee groups compared with the HF mice. Moreover, there was a significant decrease in hepatic TG levels in the HFCC and HFGC groups compared with the HF mice, whereas HFDC showed a tendency for decreased levels. There was no significant change in plasma TC in the HFDC mice, and the plasma and hepatic TC levels of all three coffee groups also tended to be lower than that of the HF group.

\section{Comparison of gene expression profiles}

We assessed the differences in muscular gene expression profiles between the $\mathrm{HF}$ and coffee groups in a DNA microarray analysis. Among the total genes analyzed, 285 genes in the HFCC mice, 247 genes in the HFDC mice, and 169 genes in the HFGC mice were differentially expressed compared with the HF mice. The list of commonly altered genes, 45 up-regulated and 17 down-regulated, is given in Supplementary Table 3.

Among the down-regulated genes, several are associated with modulating cellular stress and inflammatory response, including activating transcription factor 3 (Atf3, 0.5-fold in HFCC, 0.6-fold in HFDC, and HFGC), suppressor of cytokine signaling 3 (Socs3, 0.6-fold in HFCC, 0.5-fold in HFDC and 0.3-fold in HFGC), heat shock protein 1A (Hspala, 0.7-fold in HFCC and HFGC and 0.6-fold in HFDC), heat shock protein 1B (Hspalb, 0.6-fold in HFCC, 0.8-fold in HFDC and 0.7-fold in HFGC). In addition, an enzyme involved in fatty acid biosynthesis, stearoylcoenzyme A desaturase 1 ( $S c d 1$, 0.6-fold in HFCC, 0.7fold in HFDC and 1.1-fold in HFGC), and genes involved in the PI3K/AKT signaling encoding pathway protein
Table 1 General characteristics of the diet groups

\footnotetext{
All values are mean $\pm \mathrm{SE}$

( $n=4-5$ mice per group)

$N D$ normal diet, $H F$ high-fat diet, $H F C C$ high-fat diet with caffeinated coffee, $H F D C$ high-fat diet with decaffeinated coffee, $H F G C$ high-fat diet with unroasted caffeinated green coffee

$* p<0.05$; ** $p<0.01$ versus the HF group by Dunnett's test
}

\begin{tabular}{|c|c|c|c|c|c|}
\hline & ND & $\mathrm{HF}$ & $\mathrm{HFCC}$ & HFDC & HFGC \\
\hline Body weight (g) & $30.0 \pm 0.9^{*}$ & $39.3 \pm 1.3$ & $36.0 \pm 1.0^{*}$ & $35.2 \pm 0.1 * *$ & $35.8 \pm 0.9^{*}$ \\
\hline Average daily food intake (g) & $3.0 \pm 0.0$ & $3.0 \pm 0.0$ & $3.0 \pm 0.1$ & $2.9 \pm 0.1$ & $3.1 \pm 0.0$ \\
\hline Daily calorie intake (kcal/day) & $11.3 \pm 0.1^{* *}$ & $15.8 \pm 0.1$ & $15.6 \pm 0.5$ & $15.3 \pm 0.5$ & $16.2 \pm 0.1$ \\
\hline Mesenteric fat $(\mathrm{g})$ & $0.3 \pm 0.1^{*}$ & $0.7 \pm 0.1$ & $0.5 \pm 0.1$ & $0.4 \pm 0.0^{*}$ & $0.5 \pm 0.1$ \\
\hline Epididymal fat (g) & $0.7 \pm 0.1 * *$ & $2.3 \pm 0.2$ & $2.2 \pm 0.3$ & $2.2 \pm 0.1$ & $2.2 \pm 0.1$ \\
\hline Plasma insulin $(\mathrm{ng} / \mathrm{mL})$ & $0.2 \pm 0.0^{*}$ & $1.4 \pm 0.4$ & $1.0 \pm 0.3$ & $0.9 \pm 0.2$ & $1.3 \pm 0.2$ \\
\hline Plasma glucose $(\mathrm{ng} / \mathrm{mL})$ & $141.2 \pm 1.3$ & $172.9 \pm 2.9$ & $168.9 \pm 4.5$ & $168.9 \pm 4.0$ & $173.6 \pm 5.2$ \\
\hline Plasma TC (mg/dL) & $129.5 \pm 20.0$ & $151.8 \pm 21.8$ & $116.8 \pm 13.0$ & $153.9 \pm 10.9$ & $131.6 \pm 3.2$ \\
\hline Plasma TG (mg/dL) & $59.0 \pm 4.2^{*}$ & $73.0 \pm 3.2$ & $67.0 \pm 4.3$ & $66.0 \pm 6.0$ & $64.1 \pm 4.4^{*}$ \\
\hline Liver weight (g) & $0.9 \pm 0.0^{* *}$ & $1.1 \pm 0.0$ & $1.0 \pm 0.0$ & $1.0 \pm 0.0$ & $1.0 \pm 0.0$ \\
\hline Hepatic TC $(\mathrm{mg} / \mathrm{g})$ & $3.9 \pm 0.3$ & $3.7 \pm 0.1$ & $3.1 \pm 0.3$ & $3.6 \pm 0.2$ & $3.1 \pm 0.2$ \\
\hline Hepatic TG $(\mathrm{mg} / \mathrm{g})$ & $32.8 \pm 3.5$ & $37.9 \pm 2.9$ & $24.4 \pm 2.3^{* *}$ & $35.1 \pm 2.1$ & $26.5 \pm 2.7 *$ \\
\hline
\end{tabular}


phosphatase 2, regulatory subunit B", alpha (Ppp2r3a, 0.5fold in HFCC and HFDC and 0.7-fold in HFGC) were also downregulated. Moreover, synuclein, gamma (breast cancer-specific protein $1, S n c g$ ) which is an upstream regulator of Hspala, and Hspalb was down-regulated 0.6-fold in HFCC, 0.5 -fold in HFDC, and 0.9-fold in HFGC, and upstream regulator of Atf3, FBJ osteosarcoma oncogene (Fos) was also was decreased 0.4-fold in HFCC, 0.25-fold in HFDC, and 1.1-fold in HFGC.

In addition, genes that have been reported to be upregulated in human diabetic subjects were observed to be down-regulated in the three coffee groups, including Rasrelated associated with diabetes ( $\mathrm{Rrad}, 0.5$-fold in HFCC and HFGC, and onefold in HFDC) and secreted phosphoprotein 1 (Sppl, 0.6-fold in HFCC, 1.1-fold in HFDC and 0.9 -fold in HFGC).

On the other hand, the several up-regulated genes include protein tyrosine phosphatase, non-receptor type 11 (Ptpn11, 1.4-fold in CC, 1.6-fold in DC, and onefold in GC), and phosphodiesterase 4D interacting protein (myomegalin) (Pde4dip, 1.5-fold in CC, 1.4-fold in DC and 1.2fold in GC).
Fig. 1 Relative mRNA expression in skeletal muscle. The relative mRNA expressions of the genes Atf3, Socs3, Fos, Hspala, Hspalb, Scd1,

Ppp2r3a, Sncg, Rrad, and Spp1 were measured by RT-PCR.

Results are expressed as mean \pm SE (ND, HF: $n=4$; HFCC, HFDC, HFGC: $n=5$ ) of the fold changes normalized to $\beta$-actin mRNA expression. ${ }^{*} p<0.05$; ** $p<0.01$ versus the HF group by Dunnett's test. Corresponding to the types of coffee, CC significantly suppressed the expression of Fos, Atf3, Hspala, Hspalb, Scd1, Sncg, and Sppl and strongly decreased the expression of Rrad. Fos was significantly decreased in HFDC. HFGC significantly suppressed the expression of Fos, Atf3, Ppp2r3a, and Rrad
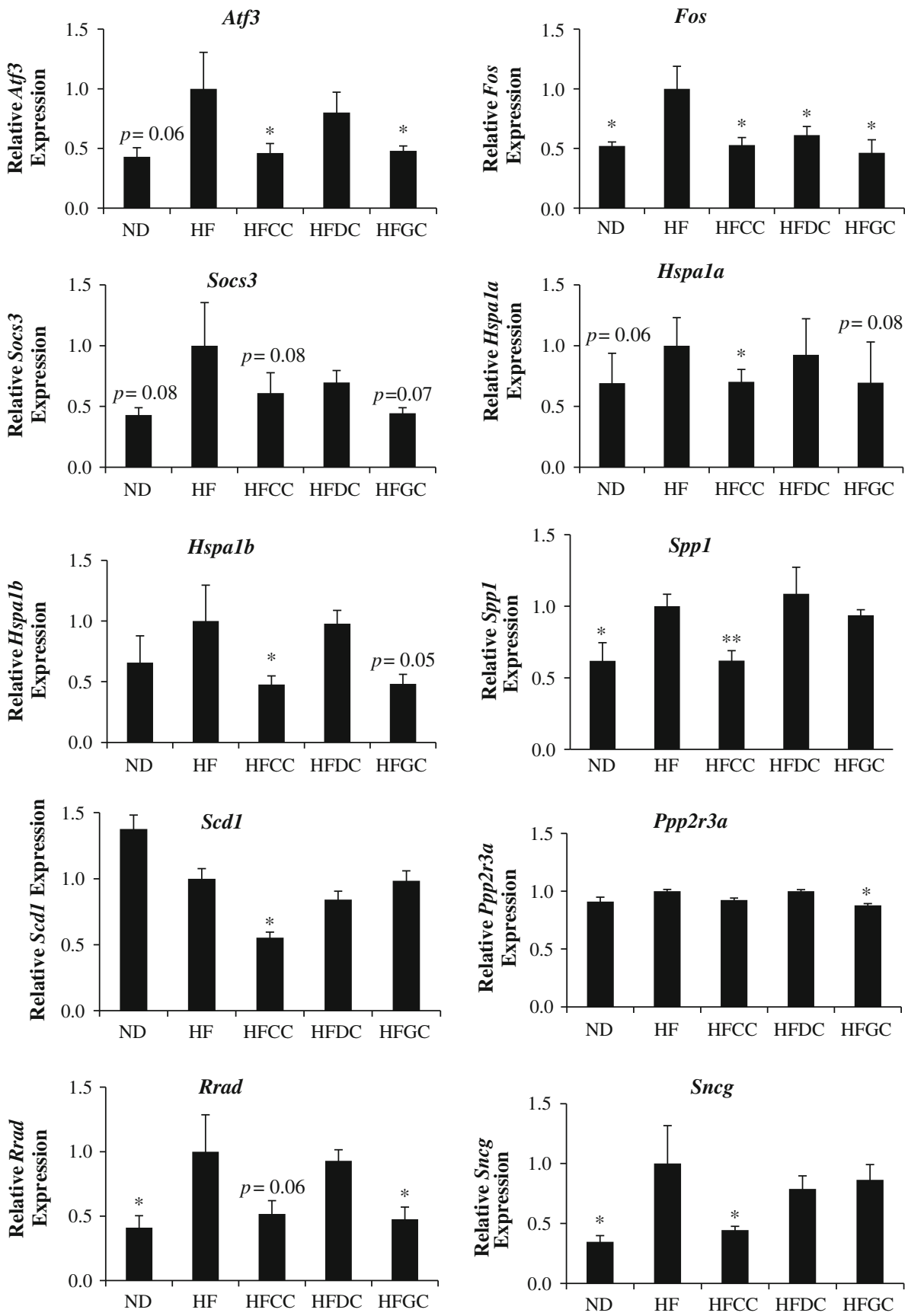
Validation of microarray results by RT-PCR

We examined the differential expression of 10 selected genes compared to the HF group by RT-PCR: Atf3, Socs3, Fos, Hspala, Hspalb, Scdl, Ppp2r3a, Sncg, Rrad, and Sppl (Fig. 1). Corresponding to the types of coffee, CC significantly suppressed the expression of Fos, Atf3, Hspala, Hspalb, Scdl, Sncg, and Sppl and strongly decreased the expression of Rrad. Fos was significantly decreased in HFDC. HFGC significantly suppressed the expression of Fos, Atf3, Ppp2r3a, and Rrad.

\section{Atf3 and Socs3 protein concentration}

Similar to the RT-PCR results, the Atf3 protein levels were significantly increased by the HF diet but significantly attenuated by the DC and GC supplementation, whereas the CC showed a strong tendency to reduce this increase (Fig. 2). In addition, the protein levels of Socs3, a downstream protein of Atf3, were significantly increased in the $\mathrm{HF}$ group, whereas a significant decrease was observed in the HFCC group, and GC strongly suppressed this increase.

\section{Insulin signaling}

The ITT was performed to elucidate the insulin signaling pathway in skeletal muscle. At $150 \mathrm{~min}$ after the insulin administration, there was a significant decrease in glucose levels in the HFGC mice compared with the HF mice, which is evident from the significantly lower $\mathrm{AUC}_{\text {glucose }}$ values in the HFGC group (Fig. 3). The glucose concentrations and $\mathrm{AUC}_{\text {glucose }}$ values of the $\mathrm{HFCC}$ and HFDC groups also tended to be lower than that of the HF group.

In addition, the western blotting analyses revealed that the insulin administration markedly elevated the tyrosine phosphorylation of IRS-1, the levels of p85/IRS-1 complex, and the serine 473 phosphorylation of Akt/PKB, most of which were suppressed in the HF group (Fig. 3). However, the tyrosine phosphorylation levels of IRS-1 tended to increase in all coffee groups compared with the HF group. The levels of the p85/IRS-1 complex were improved in the HFCC and HFGC mice, and the serine 473 phosphorylated Akt/PKB levels also tended to higher in the HFCC group.

\section{Discussion}

In the present study, we sought to assess the molecular mechanisms underlying the effects of coffee intake on impaired insulin signaling, inflammation and obesity, by conducting a comprehensive transcriptomic analysis integrated western blotting evaluation in mouse skeletal muscle, of which is the pivotal involvement in glucose
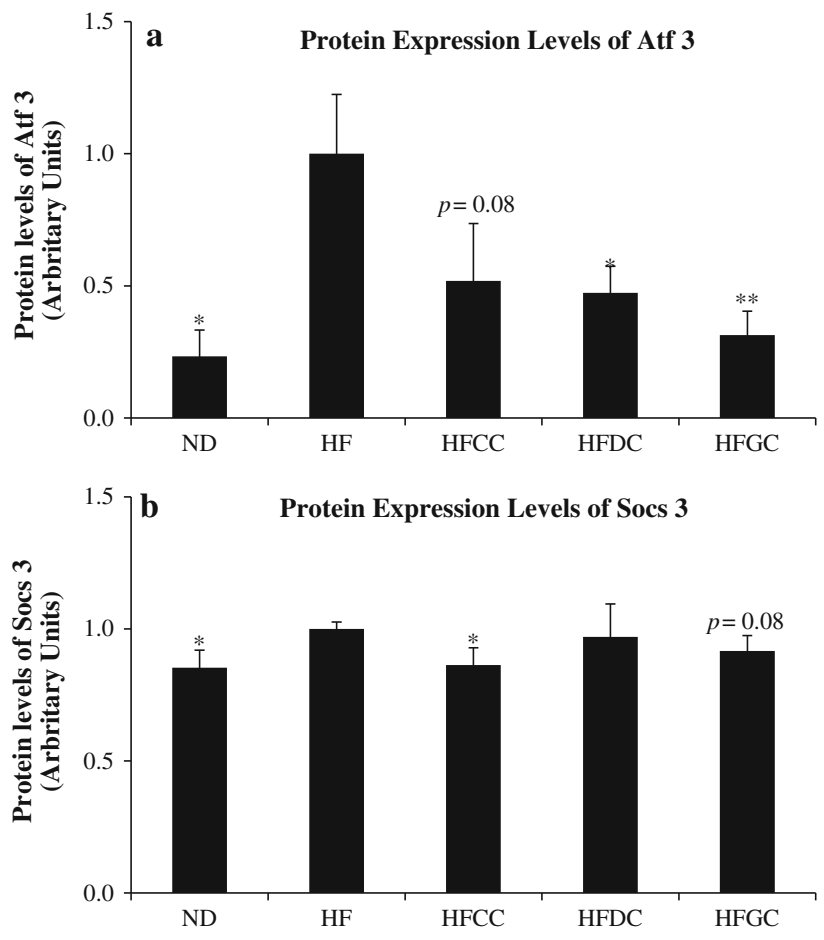

Fig. 2 Protein levels of Atf3 and Socs 3 in mouse skeletal muscle. We measured the protein levels of a Atf3 and b Socs3 in skeletal muscle using an ELISA kit. The results were normalized to the protein concentration in each sample, and the data are presented as mean \pm SE (ND, HF: $n=4$; HFCC, HFDC, HFGC: $n=5$ ) of the fold changes normalized to the protein concentration of each sample to that of the ND group. $* p<0.05 ; * * p<0.01$ versus the HF group by Dunnett's test. Atf3 protein levels were significantly increased by the HF diet but significantly attenuated by the DC and GC supplementation, whereas the $\mathrm{CC}$ showed a strong tendency to reduce this increase. Protein level of Socs3, a downstream protein of Atf3, was significantly increased in the HF group, whereas a significant decrease was observed in the HFCC group, and GC strongly suppressed this increase

metabolism and homeostasis in humans. Our results suggest that coffee intake may suppress the development of features leading to T2D.

Various dietary and lifestyle factors have been identified as contributing to the development of T2D, of which obesity has been labeled one of the most fundamental causes (Hu et al. 2001). Obesity is a systemic inflammatory response, and many inflammatory mediators exhibit expression patterns that interfere with insulin action (Pradhan 2003; Han et al. 2002; Barzilay et al. 2001; Pradhan et al. 2001). In the present study, the coffee intake by mice significantly suppressed the body weight gain and attenuated the increase in mesenteric fat weight and glucose levels during the ITT. As such, we may infer that obesity due to body weight gain and the increases in adipose tissue and insulin resistance brought about by a highfat diet may be ameliorated by coffee intake; however, further conformational studies in humans are required. 

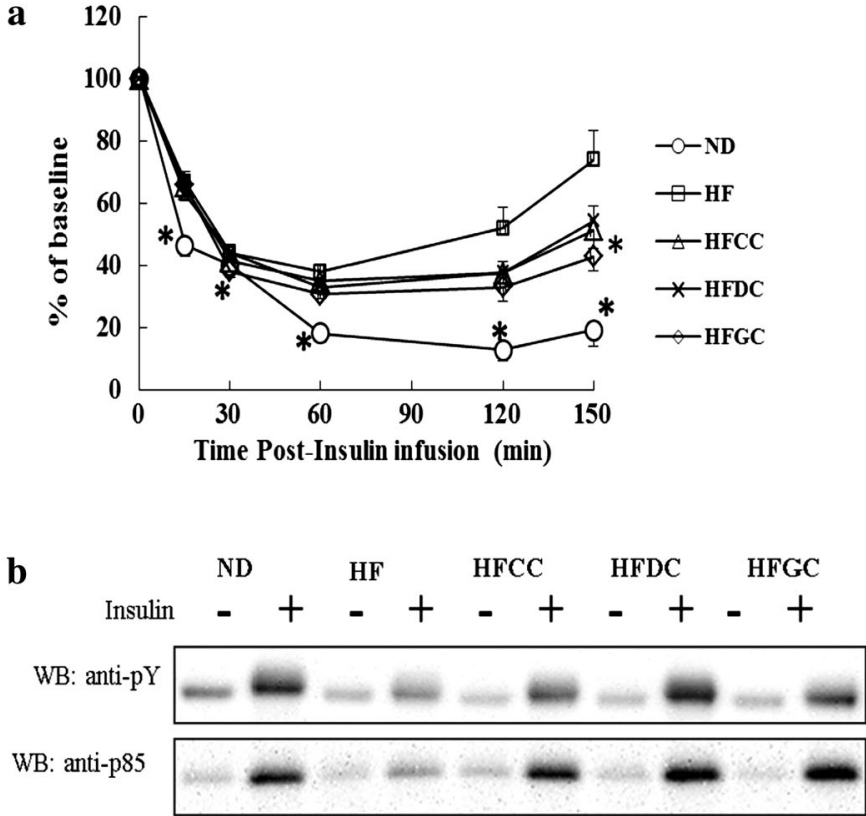

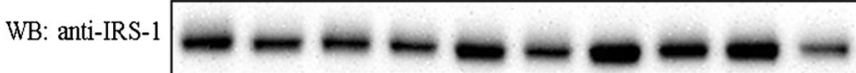

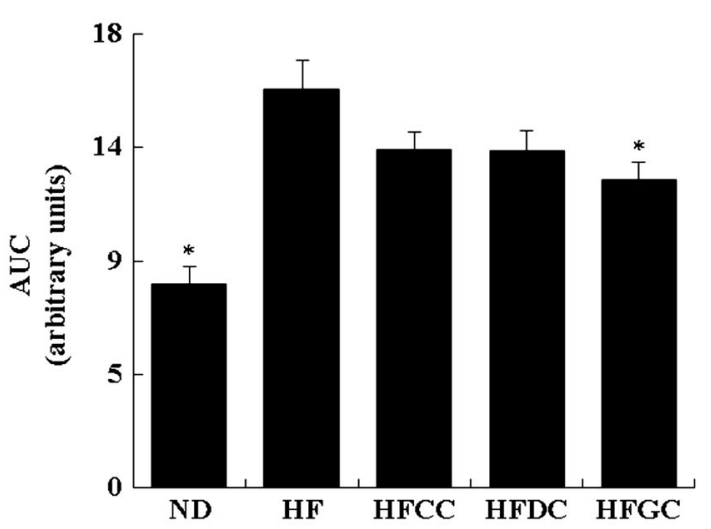

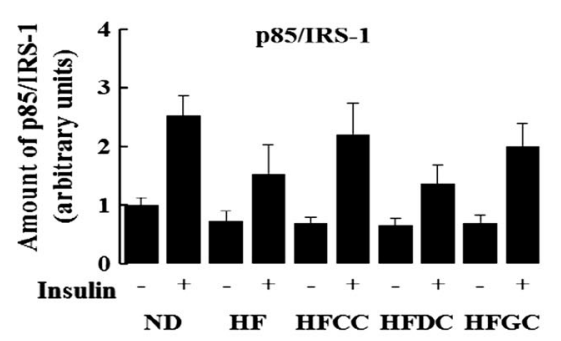

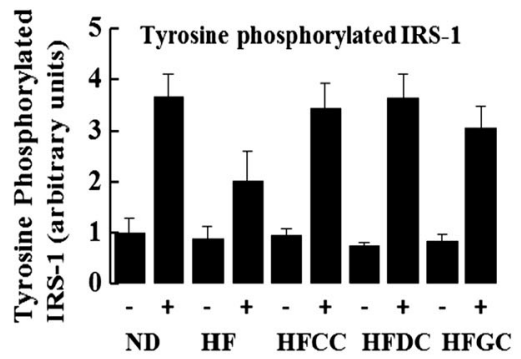

Fig. 3 Changes in glucose levels and insulin signaling during insulin stimulation. a Glucose levels and $\mathrm{AUC}_{\text {glucose }}$ during the ITT. At $150 \mathrm{~min}$ after the insulin administration, there was a significant decrease in glucose levels in the HFGC mice compared to the HF mice, which is evident from the significantly lower $\mathrm{AUC}_{\text {glucose }}$ values in the HFGC group. The glucose concentrations and $\mathrm{AUC}_{\text {glucose }}$ values of the HFCC and HFDC groups also tended to be lower than that of the HF group. b Levels of tyrosine phosphorylated IRS-1, p85/ IRS-1, and Ser473 phosphorylated Akt/PKB. The bands of immunoreactive proteins were detected with an enhanced chemiluminescence kit and quantified using an LAS-1000-plus image analyzer. Insulin

The suppression of body weight gain and insulin resistance by coffee intake was reflected in the altered expression of several genes related to inflammation, insulin signaling, and obesity. Transcription factor Atf 3 is induced by various stress signals such as high levels of glucose and fatty acids, endoplasmic reticulum stress, and reactive oxygen species (Zmuda et al. 2010) which are relevant to T2D. Atf 3 and its transcription factor, Fos, have been found to be up-regulated in the skeletal muscle of insulin-resistant humans, where prolonged activation of proinflammatory pathways within insulin target cells which leads to obesity-

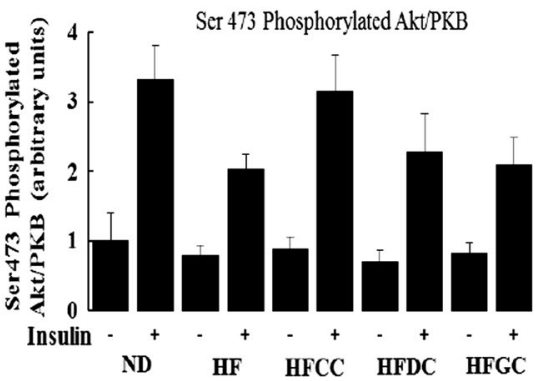

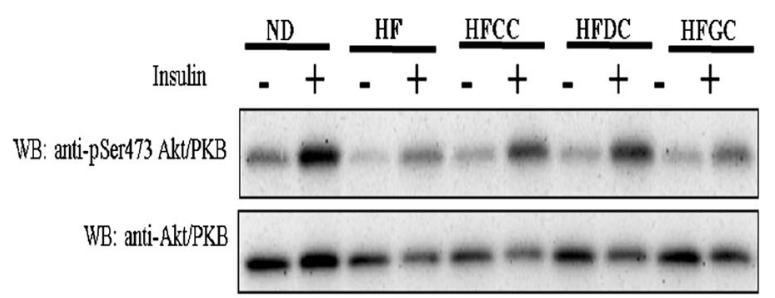

administration markedly elevated the tyrosine phosphorylation of IRS-1, the levels of p85/IRS-1 complex, and the serine473 phosphorylation of $\mathrm{Akt} / \mathrm{PKB}$, most of which were suppressed in the HF group. However, the tyrosine phosphorylation levels of IRS-1 tended to increase in all coffee groups compared to the HF group. The levels of the p85/IRS-1 complex were improved in the HFCC and HFGC mice, and the serine 473 phosphorylated Akt/PKB levels also tended to higher in the HFCC group. All data are presented as mean $\pm \mathrm{SE}$ (ND, HF: $n=4$; HFCC, HFDC, HFGC: $n=5$ ) of the fold changes normalized to the levels of each sample to that of the ND group. ${ }^{*} p<0.05 ; * *<0.01$ versus the HF group by Dunnett's test

related insulin resistance can be attributed to the activation of the JNK/activator protein 1 (AP1) signaling pathways composed of c-Jun and c-Fos proteins (Bossis et al. 2005). Moreover, a downstream target gene of Atf3, Socs 3 which is a major negative regulator of both leptin and insulin signaling, (Jiang et al. 2004) impairs muscle insulin signaling and promotes systemic insulin resistance (Jiang et al. 2004). In the present study, the elevated expressions of Atf3, Fos and Socs 3 in the mice fed an HF diet were reduced by coffee intake. In particular, CC and GC may improve insulin signaling in skeletal muscle, partially via 
the increased tyrosine phosphorylation of IRS-1 due to lowered Socs 3 expression.

The Atf3 protein levels were also decreased in all three of the present coffee groups, with substantial decreases in the mice fed the HFDC and HFGC diets. CGA, a component of DC and GC, has been reported to exert antiinflammatory effects via the attenuation of the activation of JNK/AP1 pathways (Shan et al. 2009). As CGA is abundant in DC and GC (Supplementary Table 1), we may infer that CGA may contribute to the reduction in Atf3 protein levels observed in the present HFDC and HFGC groups. Moreover, we can also deduce that the anti-inflammatory properties of CA which exists in high concentrations in CC and GC (Supplementary Table 1) may play a role in the reduced expression of Atf3, Fos and Socs3. This idea is also supported by the increased levels of p85/IRS-1 observed only in the caffeine-consuming mice.

The expression levels of $S c d l$ induced by Atf 3 were also similarly increased in the HF group and significantly reduced in the HFCC group. $S c d l$ encodes for the microsomal enzyme stearoyl-CoA desaturase 1 , which is involved in the conversion of saturated fatty acids (Peter et al. 2009). High SCD1 inducibility was associated with ATF3 expression, and this was positively correlated with intramyocellular lipids and insulin sensitivity (Peter et al. 2009). Another gene similar to Scd1 with an effect on inflammation-associated insulin signaling is Sppl. Sppl, a multifunctional protein, has an important role in a variety of inflammatory disorders, e.g., atherosclerosis, and in diabetic macro- and micro-vascular diseases and hepatic inflammation where the antibody-mediated neutralization of Spp1 in obese mice was reported to rapidly improve insulin sensitivity (Kiefer et al. 2010). In the present study, the CC significantly attenuated the increases in $S c d l$ and Sppl expression induced by the HF diet, and the improvement in inflammation-associated insulin signaling was reflected by the increase in the tyrosine phosphorylation of IRS-1, p85/IRS-1 complex and Ser473 phosphorylated Akt/PKB levels and lower insulin levels.

Hspala and Hspalb are two copies of genetically similar genes encoding heat shock protein 70 (Hsp70) (Smith et al. 2007) usually elevated during high levels of oxidative stress and glucose deprivation and have been implicated in immune-mediated type 1 diabetes, insulin resistance, and obesity-related T2D (Smith et al. 2007). The up-regulation of Hspala and Hspalb in the present HF group compared to the ND controls indicates an inflammatory state brought about by the high-fat diet. The expression levels of these genes were significantly lower in the HFCC group and tended to be lower in HFGC group but were not changed in the HFDC group, suggesting that $\mathrm{CA}$ and CGA which exist in high concentrations in $\mathrm{CC}$ and $\mathrm{GC}$ might play an important role in reducing obesity-related inflammatory and oxidative insults (Chao et al. 2009; Jung 2006).

In addition, Sncg, a novel chaperone in the expression of Hsp70, encodes for the gamma-synuclein as a marker of peripheral and selects central nervous system neurons (Oort et al. 2008). Compromised peripheral nervous system function in obesity, prediabetes, and T2D is an early event accompanying system-wide metabolic dysregulation (Oort et al. 2008). To our knowledge, our present finding is the first evidence that $S n c g$ expression in skeletal muscle was elevated in high-fat diet-induced obese mice, suggesting that significant attenuation in Sncg expression levels in CC may revive compromised neural signaling other than that in adipose tissue to improve system-wide metabolic dysregulation.

The expression level of Rrad, an inhibitor of voltagegated calcium channels, was reported to be associated with the accumulation of reactive oxygen species (Halter et al. 2010), and more importantly, Rrad was identified as being overexpressed in the skeletal muscle of T2D patients and diabetic rats (Reynet and Kahn 1993). Another gene that is up-regulated in diabetic patients is Ppp2r3a, which encodes for serine/threonine specific protein phosphatase 2 (also known as protein phosphatase 2A, PP2A) (Højlund et al. 2002; Mumby and Walter 1993) which dephosphorylates a number of enzymes in the insulin signaling cascade believed to regulate glucose transport and glycogen synthesis. In the present study, the HFCC and HFGC diets suppressed the up-regulation of Rrad expression, and the HFGC diet attenuated the increase in Ppp2r3a expression. CA was also reported to lower plasma glucose levels (Jung 2006), from which we may infer that CA which is abundant in CC and GC may contribute to the reduction in Rrad expression. In addition, the lowered levels of Ppp2r3a in the HFGC mice corresponded to the mediated insulin signaling observed from an increase in the tyrosine phosphorylation of IRS-1 and p85/IRS-1 complex.

In much of the world, the rising incidence of obesity demands novel preventive and therapeutic approaches to the treatment of obesity-associated complications-particularly insulin resistance-that lead to T2D and promote cardiovascular disease. Chronic over-nutrition leading to obesity (Esposito 2002; Mohanty et al. 2000) has resulted in elevated levels of inflammatory markers which might interfere with insulin action by suppressing insulin signal transduction, resulting in insulin resistance, which in turn promotes inflammation (Dandona et al. 2001; Aljada et al. 2002). In the present study, coffee intake exerted its antiinflammatory properties in pathways related to the downregulation of Atf3, Fos, Socs3, Hspala, Hspalb, and Sncg. Mediated inflammation-associated insulin signaling was also partly reflected in the decreased expressions of $S c d l$ 
Fig. 4 Schematic representation of the metabolic changes produced by $\mathrm{CC}, \mathrm{DC}$, and GC in the skeletal muscle of high-fat diet-induced obese mice. Letters in light green had a strong tendency whereas letters in dark green indicate a significant improvement mediated by coffee consumption

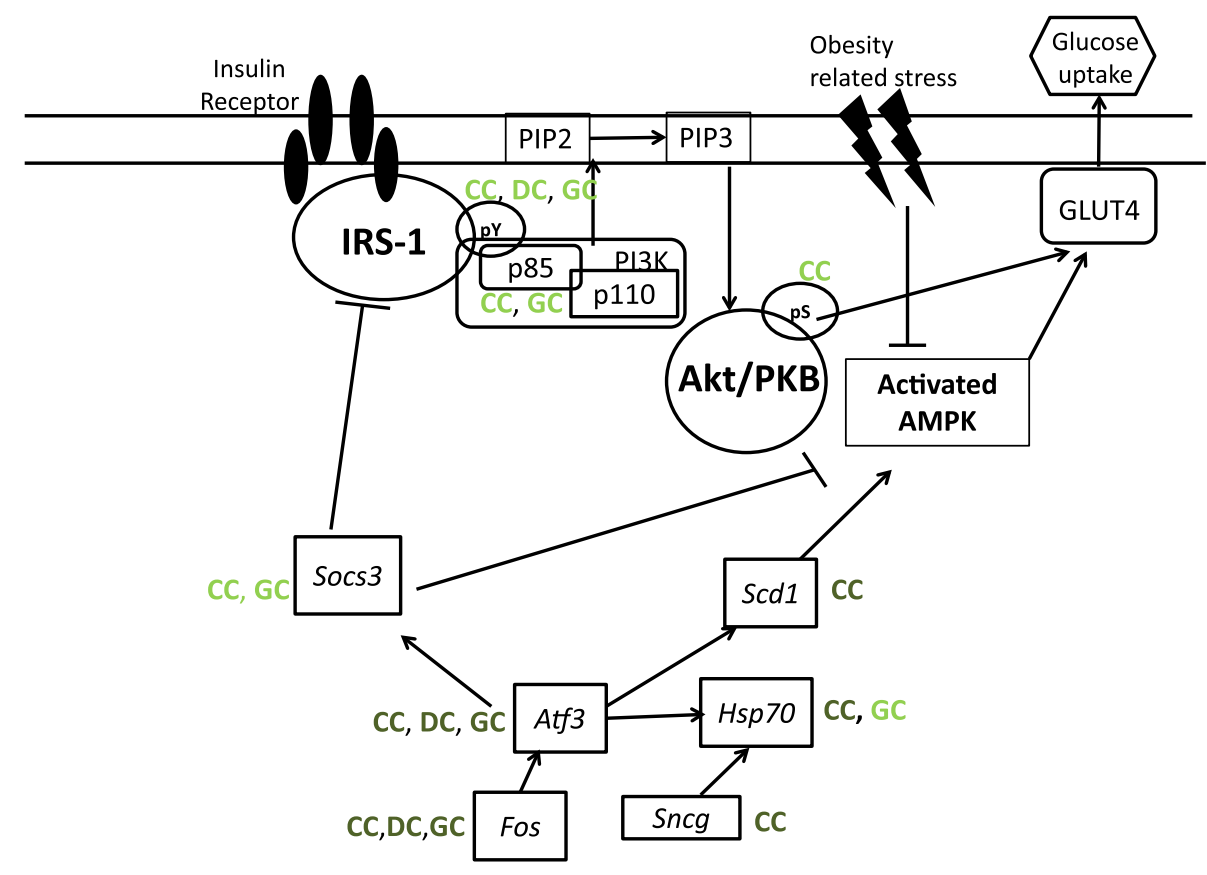

and Spp1. In addition, the up-regulation of Rrad and Ppp2r3a by the HF diet, which is similar to the up-regulation seen in diabetic patients, was improved by coffee consumption. According to the dose translation from mouse studies to human (Reagan-Shaw et al. 2007), the $2 \%$ coffee supplementation used in this study is equivalent to 4 cups of coffee per day, in a $60-\mathrm{kg}$ human being, a daily dosage which is not difficult to achieve.

As illustrated in Fig. 4, the coffee intake also mitigated the insulin signaling to some extent in skeletal muscle by lowering insulin concentrations during the ITT, increasing the tyrosine phosphorylation of IRS-1, increasing the levels of the p85/IRS- 1 complex and up-regulating the serine 473 phosphorylation of Akt/PKB. As mentioned above, not only have our findings highlighted the anti-inflammatory properties of coffee intake, we have also ascertained several genes which may improve the insulin resistance brought about by high-fat diet-induced obesity through a transcriptomic evaluation. In this study, we have elucidated the possible molecular mechanisms underlying the beneficial effects of coffee intake which suggests that coffee intake may play a part in the prevention of obesity, obesityassociated insulin resistance in skeletal muscle and T2D. Based on the findings of this study, we have provided scientific evidence that suggest coffee intake may be effective as a dietary intervention in obesity management and the prevention of T2D.

Acknowledgments This study was partly supported by a grant from the All Japan Coffee Association.

Conflict of interest All the authors declare no conflict of interest.

\section{References}

Abebe A, Kebba S (2013) Determination of chlorogenic acids (CGA) in coffee beans using HPLC. Am J Res Commun 1(2):78-91

Aljada A, Ghanim H, Mohanty P, Kapur N, Dandona P (2002) Insulin inhibits the pro-inflammatory transcription factor early growth response gene-1 (Egr)-1 expression in mononuclear cells (MNC) and reduces plasma tissue factor (TF) and plasminogen activator inhibitor-1 (PAI-1) concentrations. J Clin Endricrinol Metab 87(3):1419-1422

Barzilay JI, Abraham L, Heckbert SR, Cushman M, Kuller LH, Resnick HE, Tracy RP (2001) The relation of markers of inflammation to the development of glucose disorders in the elderly the Cardiovascular Health Study. Diabetes 50(10):2384-2389

Bossis G, Malnou CE, Farras R, Andermarcher E, Hipskind R, Rodriguez M, Schmidt D, Muller S, Jariel-Encontre I, Piechaczyk M (2005) Down-regulation of c-Fos/c-Jun AP-1 dimer activity by sumoylation. Mol Cell Biol 25(16):6964-6979. doi:10.1128/mcb.25.16.6964-6979.2005

Chao PC, Hsu CC, Yin MC (2009) Anti-inflammatory and anticoagulatory activities of caffeic acid and ellagic acid in cardiac tissue of diabetic mice. Nutr Metab 6(1):33. doi:10.1186/17437075-6-33

Cho AS, Jeon SM, Kim MJ, Yeo J, Seo KI, Choi MS, Lee MK (2010) Chlorogenic acid exhibits anti-obesity property and improves lipid metabolism in high-fat diet-induced-obese mice. Food Chem Toxicol 48(3):937-943. doi:10.1016/j.fct.2010.01.003

Dandona P, Aljada A, Mohanty P, Ghanim H, Hamouda W, Assian E, Ahmad S (2001) Insulin inhibits intranuclear nuclear factor kappaB and stimulates IkappaB in mononuclear cells in obese subjects: evidence for an anti-inflammatory effect? J Clin Endricrinol Metab 86(7):3257-3265

DeFronzo RA, Bonadonna RC, Ferrannini E (1992) Pathogenesis of NIDDM. Diabetes Care 15(3):318-368

Esposito K (2002) Inflammatory cytokine concentrations are acutely increased by hyperglycemia in humans: role of oxidative stress. Circulation 106(16):2067-2072. doi:10.1161/01.cir.0000034509. 14906.ae 
Gray J (1998) Caffeine, coffee and health. Nutr Food Sci 98(6):314-319

Halter B, Gonzalez de Aguilar J-L, Rene F, Petri S, Fricker B, Echaniz-Laguna A, Dupuis L, Larmet Y, Loeffler J-P (2010) Oxidative stress in skeletal muscle stimulates early expression of Rad in a mouse model of amyotrophic lateral sclerosis. Free Radic Biol Med 48(7):915-923. doi:10.1016/j.freeradbiomed. 2010.01.014

Han TS, Sattar N, Williams K, Gonzalez-Villalpando C, Lean ME, Haffner SM (2002) Prospective study of C-reactive protein in relation to the development of diabetes and metabolic syndrome in the Mexico City Diabetes Study. Diabetes Care 25(11):2016-2021

Højlund K, Poulsen M, Staehr P, Brusgaard K, Beck-Nielsen H (2002) Effect of insulin on protein phosphatase 2A expression in muscle in type 2 diabetes. Eur J Clin Investig 32(12):918-923

Hu FB, Manson JE, Stampfer MJ, Colditz G, Liu S, Solomon CG, Willett WC (2001) Diet, lifestyle, and the risk of type 2 diabetes mellitus in women. N Engl J Med 345(11):790-797

Hu G, Jousilahti P, Peltonen M, Bidel S, Tuomilehto J (2006) Joint association of coffee consumption and other factors to the risk of type 2 diabetes: a prospective study in Finland. Int $\mathrm{J}$ Obes 30(12):1742-1749. doi:10.1038/sj.ijo.0803341

Jia H, Saito K, Aw W, Takahashi S, Hanate M, Hasebe Y, Kato H (2013a) Transcriptional profiling in rats and an ex vivo analysis implicate novel beneficial function of egg shell membrane in liver fibrosis. J Funct Foods 5(4):1611-1619. doi:10.1016/j.jff. 2013.07.003

Jia H, Takahashi S, Saito K, Kato H (2013b) DNA microarray analysis identified molecular pathways mediating the effects of supplementation of branched-chain amino acids on CCl4induced cirrhosis in rats. Mol Nutr Food Res 57(2):291-306. doi: $10.1002 / \mathrm{mnfr} .201200538$

Jiang HY, Wek SA, McGrath BC, Lu D, Hai T, Harding HP, Wang X, Ron D, Cavener DR, Wek RC (2004) Activating transcription factor 3 is integral to the eukaryotic initiation factor 2 kinase stress response. Mol Cell Biol 24(3):1365-1377. doi:10.1128/ mcb.24.3.1365-1377.2004

Jung UJ (2006) Antihyperglycemic and antioxidant properties of caffeic acid in db/db mice. J Pharmacol Exp Ther 318(2): 476-483. doi:10.1124/jpet.106.105163

Kiefer FW, Zeyda M, Gollinger K, Pfau B, Neuhofer A, Weichhart T, Saemann MD, Geyeregger R, Schlederer M, Kenner L, Stulnig TM (2010) Neutralization of osteopontin inhibits obesityinduced inflammation and insulin resistance. Diabetes 59(4): 935-946. doi:10.2337/db09-0404

Kobayashi M, Matsuda Y, Iwai H, Hiramitsu M, Inoue T, Katagiri T, Yamashita Y, Ashida H, Murai A, Horio F (2012) Coffee improves insulin-stimulated Akt phosphorylation in liver and skeletal muscle in diabetic KK-A(y) mice. J Nutr Sci Vitaminol 58(6):408-414

Matsuda Y, Kobayashi M, Yamauchi R, Ojika M, Hiramitsu M, Inoue T, Katagiri T, Murai A, Horio F (2011) Coffee and caffeine improve insulin sensitivity and glucose tolerance in C57BL/6J mice fed a high-fat diet. Biosci Biotechnol Biochem 75(12):2309-2315. doi:10.1271/bbb. 110452

Mohanty P, Hamouda W, Garg R, Aljada A, Ghanim H, Dandona P (2000) Glucose challenge stimulates reactive oxygen species (ROS) generation by leucocytes. J Clin Endricrinol Metab 85(8):2970-2973

Mumby MC, Walter G (1993) Protein serine/threonine phosphatases: structure, regulation, and functions in cell growth. Physiol Rev 73(4):673-699
Nardini M, D’Aquino M, Tomassi G, Gentili V, Di Felice M, Scaccini C (1995) Inhibition of human low-density lipoprotein oxidation by caffeic acid and other hydroxycinnamic acid derivatives. Free Radic Biol Med 19(5):541-542

Oort PJ, Knotts TA, Grino M, Naour N, Bastard JP, Clément K, Ninkina N, Buchman VL, Permana PA, Luo X, Pan G, Dunn TN, Adams SH (2008) Gamma-synuclein is an adipocyte-neuron gene coordinately expressed with leptin and increased in human obesity. J Nutr 138(5):841-848

Paynter NP, Yeh HC, Voutilainen S, Schmidt MI, Heiss G, Folsom AR, Brancati FL, Kao WHL (2006) Coffee and sweetened beverage consumption and the risk of type 2 diabetes mellitus: the atherosclerosis risk in communities study. Am J Epidemiol 164(11):1075-1084. doi:10.1093/aje/kwj323

Peter A, Weigert C, Staiger H, Machicao F, Schick F, Machann J, Stefan N, Thamer C, Haring HU, Schleicher E (2009) Individual stearoyl-CoA desaturase 1 expression modulates endoplasmic reticulum stress and inflammation in human myotubes and is associated with skeletal muscle lipid storage and insulin sensitivity in vivo. Diabetes 58(8):1757-1765. doi:10.2337/ db09-0188

Pradhan AD (2003) C-reactive protein is independently associated with fasting insulin in nondiabetic women. Arterioscler Thromb Vasc Biol 23(4):650-655. doi:10.1161/01.atv.0000065636. $15310.9 \mathrm{c}$

Pradhan AD, Manson JE, Rifai N, Buring JE, Ridker PM (2001) C-reactive protein, interleukin 6, and risk of developing type 2 diabetes mellitus. JAMA 286(3):327-334

Reagan-Shaw S, Nihal M, Ahmad N (2007) Dose translation from animal to human studies revisited. FASEB J 22(3):659-661. doi:10.1096/fj.07-9574LSF

Reynet C, Kahn CR (1993) Rad: a member of the Ras family overexpressed in muscle of type II diabetic humans. Science 262(5138): 1441-1444

Rice-Evans CA, Miller NJ, Paganga G (1996) Structure-antioxidant activity relationships of flavonoids and phenolic acids. Free Radic Biol Med 20(7):933-956

Shan J, Fu J, Zhao Z, Kong X, Huang H, Luo L, Yin Z (2009) Chlorogenic acid inhibits lipopolysaccharide-induced cyclooxygenase-2 expression in RAW264.7 cells through suppressing NF- $\kappa \mathrm{B}$ and JNK/AP-1 activation. Int Immunopharmacol 9(9):1042-1048. doi:10.1016/j.intimp.2009.04.011

Smith R, Meyers D, Peters S, Moore W, Wenzel S, Bleecker E, Hawkins G (2007) Sequence analysis of HSPA1A and HSPA1B in a multi-ethnic study population. DNA Seq 18(1):47-53. doi:10.1080/10425170601060707

Stefano P (2002) The latte revolution regulation markets and consumption in the global coffee chain. World Dev 30(7): $1099-1122$

Takahashi S, Egashira K, Saito K, Jia H, Abe K, Kato H (2014) Coffee intake down-regulates the hepatic gene expression of peroxisome proliferator-activated receptor gamma in C57BL/6J mice fed a high-fat diet. J Funct Foods 6:157-167. doi:10.1016/j. jff.2013.10.002

Zmuda EJ, Qi L, Zhu MX, Mirmira RG, Montminy MR, Hai T (2010) The roles of ATF3, an adaptive-response gene, in high-fat-dietinduced diabetes and pancreatic -cell dysfunction. Mol Endocrinol 24(7):1423-1433. doi:10.1210/me.2009-0463 